\title{
Epidermólise Bolhosa: manifestações orais e manejo odontológico
}

\author{
Epidermolysis Bullosa: oral manifestations and dental management
}

Epidermólisis ampollosa: manifestaciones bucales y tratamiento dental

Marcela Nogueira dos Santos ORCID: https://orcid.org/0000-0002-0554-6382

Faculdade de Odontologia do Recife, Brasil

E-mail: marcelaanogueira.santos@gmail.com

Amanda Gonçalves Franco

ORCID: https://orcid.org/0000-0003-0983-7539 Universidade de Itaúna, Brasil

E-mail: amandagfranco38@gmail.com

Daniele Saara dos Santos

ORCID: https://orcid.org/0000-0001-9381-5580

Universidade Federal de Pernambuco, Brasil

E-mail: saaaradaniele@gmail.com

Wenys Cláudio Gomes da Silva ORCID: https://orcid.org/0000-0002-9321-183X

Universidade Federal de Pernambuco, Brasil

E-mail: wenys.claudio@ufpe.br

Lecy Anne Braga Gomes

ORCID: https://orcid.org/0000-0002-2398-6488

Universidade Evangélica de Goiás, Brasil

E-mail: lele_bg14@hotmail.com

Alexandre de Souza Miranda

ORCID: https://orcid.org/0000-0002-1608-7636

Universidade Evangélica de Goiás, Brasil

E-mail: alexandremiranda1_@outlook.com

Marcelo Costa Rodrigues

ORCID: https://orcid.org/0000.0002-6606-0538

Universidade Federal de Jatai, Brasil

E-mail: rodriguesmc17@gmail.com

Grace Kelly Martins Carneiro

ORCID: https://orcid.org/0000-0001-6679-8930

Faculdade Morgana Potrich, Brasil

E-mail: carneirogkm.gc@gmail.com

Thallita Monalisa Sizenando Souza Lima

ORCID: https://orcid.org/0000-0003-1224-9420

Universidade Federal do Rio Grande do Norte, Brasil

E-mail: monalisa.sizenando.094@ufrn.edu.br

Nívia Delamoniky Lima Fernandes

ORCID: https://orcid.org/0000-0001-8439-2117

Centro Universitário Inta, Brasil

E-mail: delamonikynivia@gmail.com

Jefferson Douglas Lima Fernandes

ORCID: https://orcid.org/0000-0002-5231-3813

Universidade Federal do Ceará, Brasil

E-mail: jefferson.odonto97@gmail.com

Matheus Almeida Ramalho

ORCID: https://orcid.org/0000-0002-5607-1313

Universidade Federal de Jataí, Brasil

E-mail: matheusramalho@discente.ufg.br

Jaqueline Lopes Reis

ORCID: https://orcid.org/0000-0003-1685-0336

Universidade de Rio Verde, Brasil

E-mail: jaquelreis@hotmail.com

Vitória Ribeiro Barbosa de Menezes

ORCID: https://orcid.org/0000-0001-6750-4770

Centro Universitário Maurício de Nassau, Brasil

E-mail: vickymenezes1999@gmail.com Jamille de Souza Castro

ORCID: https://orcid.org/0000-0003-3257-8651

Centro Universitário Alfredo Nasser, Brasil

E-mail: castrosjamille@gmail.com 


\author{
Áquila de Oliveira Afonso \\ ORCID: https://orcid.org/0000-0003-2392-4022 \\ Universidade Evangélica de Goiás, Brasil \\ E-mail: aquilaafonso1@gmail.com
}

\begin{abstract}
Resumo
A Epidermólise Bolhosa (EB) descreve um grupo de doenças rara, de caráter hereditário, caracterizada pelo desenvolvimento de bolhas na região cutaneomucosa de todo o corpo, em resposta ao trauma mínimo, ao calor, ou a nenhuma causa aparente, podendo manifestar-se ao nascimento ou durante os primeiros anos de vida. o presente estudo possui como objetivo revisar a literatura acerca das manifestações orais encontradas em pacientes portadores da Epidermólise Bolhosa, bem como descrever o manejo clínico odontológico a ser seguido em casos de EB. Para a construção deste trabalho foi feito um levantamento bibliográfico nas bases de dados SciVerse Scopus, Scientific Eletronic Library Online (Scielo), U.S. National Library of Medicine (PUBMED) e ScienceDirect, utilizando o gerenciador de referências Mendeley. Na cavidade oral, a EB pode manifestar-se de diversas formas, com inúmeras alterações, que variam em severidade e quantidade, dependendo do tipo de EB, como a formação de bolhas e ulcerações (na mucosa, palato e língua), anodontia, junção do sulco vestibular (união da gengiva inserida com a mucosa bucal), presença das doenças cárie e periodontal severas, reabsorção óssea alveolar e anomalias dentárias hipoplásicas, além de atrofia da maxila, prognatismo mandibular e ângulo da mandíbula aumentado. A maloclusão torna-se ainda mais severa pela atresia dos arcos alveolares e pelo atraso na erupção dentária. Devido à grande dificuldade e risco de se oferecer tratamento odontológico aos pacientes com EB, a prevenção da cárie, biofilme e da doença periodontal deve ser enfatizada desde a infância. Devendo o profissional no tratamento realizar higiene oral e profilaxia adequada, um programa dietético de prevenção de cárie, uso de géis, enxaguantes bucais, terapias adjuvantes com clorexidina $\mathrm{D}$, cotonetes, sprays e aplicações de verniz tópico, fluoretos, raspagem de biofilme e lazer.
\end{abstract}

Palavras-chave: Manejo odontológico; Manifestações orais; Epidermólise bolhosa.

\begin{abstract}
Epidermolysis Bullosa (EB), a group of rare hereditary diseases, characterized by the development of blisters in the cutaneous-mucosal region of the entire body, in response to minimal trauma, heat, or no apparent cause. birth or during the first years of life. The present study aims to review the literature on the oral manifestations found in patients with Epidermolysis Bullosa, as well as to define the dental clinical management to be followed in cases of EB. SciVerse Scopus, Scientific Electronic Library Online (Scielo), US National Library of Medicine (PUBMED) and ScienceDirect, using the Mendeley Reference Manager. In the oral cavity, an EB can manifest itself in several ways, with changes that vary in severity and quantity, depending on the type of EB, such as the formation of blisters and ulcerations (in the mucosa, palate and tongue), anodontia, junction of the vestibular sulcus (union of the attached gingiva with the oral mucosa), presence of caries and severe periodontal diseases, alveolar bone resorption and hypoplastic dental anomalies, in addition to maxillary atrophy, mandibular prognathism and mandibular course. Malocclusion is even more severe due to atresia of the alveolar arches and delay in tooth eruption. Due to the great difficulty and risk of offering dental treatment to patients with EB, the prevention of disease, biofilm and periodontal disease must be emphasized from childhood. Professionals in treatment should carry out oral hygiene and adequate prophylaxis, a diet program to prevent caries, use of gels, mouthwashes, adjuvant therapies with chlorhexidine D, cotton swabs, sprays and topical varnish applications, fluorides, biofilm scraping and leisure.
\end{abstract}

Keywords: Dental management; Oral manifestations; Bullous epidermolysis.

\title{
Resumen
}

Epidermólisis ampollosa (EB), grupo de enfermedades hereditarias raras, caracterizadas por el desarrollo de ampollas en la región cutáneo-mucosa de todo el cuerpo, en respuesta a traumatismos mínimos, calor o sin causa aparente. Nacimiento o durante los primeros años de vida. vida. El presente estudio tiene como objetivo revisar la literatura sobre las manifestaciones bucales encontradas en pacientes con Epidermólisis Bullosa, así como definir el manejo clínico dental a seguir en los casos de EB. SciVerse Scopus, Scientific Electronic Library Online (Scielo), Biblioteca Nacional de Medicina de EE. UU. (PUBMED) y ScienceDirect, utilizando el Administrador de referencias de Mendeley. En la cavidad oral, una EB puede manifestarse de varias formas, con cambios que varían en gravedad y cantidad, según el tipo de EB, como la formación de ampollas y ulceraciones (en la mucosa, paladar y lengua), anodoncia, unión del surco vestibular (unión de la encía adherida con la mucosa oral), presencia de caries y enfermedades periodontales graves, reabsorción ósea alveolar y anomalías dentales hipoplásicas, además de atrofia maxilar, prognatismo mandibular y curso mandibular. La maloclusión es aún más grave debido a la atresia de los arcos alveolares y al retraso de la erupción dentaria. Debido a la gran dificultad y riesgo de ofrecer tratamiento odontológico a pacientes con EB, se debe enfatizar la prevención de enfermedades, biofilm y enfermedad periodontal desde la niñez. Los profesionales en tratamiento deben realizar higiene bucal y profilaxis adecuada, un programa de dieta para prevenir caries, uso de geles, enjuagues bucales, terapias adyuvantes con clorhexidina $\mathrm{D}$, hisopos de algodón, aerosoles y aplicaciones de barnices tópicos, fluoruros, raspado de biofilm y ocio.

Palabras clave: Manejo dental; Manifestaciones orales; Epidermólisis ampollosa. 


\section{Introdução}

A Epidermólise Bolhosa (EB) descreve um grupo de doenças rara, de caráter hereditário, caracterizada pelo desenvolvimento de bolhas na região cutaneomucosa de todo o corpo, em resposta ao trauma mínimo, ao calor, ou a nenhuma causa aparente, podendo manifestar-se ao nascimento ou durante os primeiros anos de vida (Angelo et al. 2012).

As bolhas variam, a depender do caso, de superficiais até mais profundas, ocorrendo seu aumento de tamanho, podendo ser dolorosas e com sangue, visto que a separação das camadas localizam-se abaixo da junção da epiderme com a derme, onde se encontram vasos sanguíneos e nervos, além de haver caimento das unhas e feridas que podem evoluir para infecções ou deixar cicatrizes que levam a complicações como pseudosindactilia e distrofia das mãos, causando a perda dos movimentos dos dedos, dificultando a socialização desse paciente com o meio em que vive (Bega et al., 2015).

A EB pode ser adquirida ou hereditária. Na sua forma adquirida, a doença manifesta-se, normalmente, na fase adulta, na qual são observados depósitos de imunoglobulina $\mathrm{G}$ no tecido localizado abaixo da membrana basal, caracterizando a epidemólise bolhosa como autoimune. Já em sua forma hereditária, a EB a é subdividida em quatro tipos principais de EB: EB simples (EBS), EB juncional (JEB), EB distrófico (DEB) e síndrome de Kindler, bem como vários subtipos (Pitta et al. 2016). A tabela 1 aborda os tipos básicos encontrados na EB.

Tabela 1. Classificação da Epidermólise bolhosa.

\begin{tabular}{cl}
\hline \multicolumn{1}{c}{ Tipos } & \multicolumn{1}{c}{ Características } \\
\hline EB Simples (EBS) & $\begin{array}{l}\text { A formação das bolhas é superficial e não deixa cicatrizes. O surgimento das bolhas } \\
\text { diminui com a idade. }\end{array}$ \\
\hline EB Distrófica (EBD) & $\begin{array}{l}\text { As bolhas são profundas e se formam abaixo da epiderme, na derme, abaixo da } \\
\text { membrana basal, o que leva a cicatrizes e muitas vezes perda da função do membro. É a } \\
\text { forma que deixa mais sequelas. }\end{array}$ \\
\hline EB Juncional (EBJ) & $\begin{array}{l}\text { Bolhas profundas. Acometem grande parte da superfície corporal, tornando-se mais } \\
\text { grave. Uma vez controladas as complicações, a doença tende a melhorar com a idade. }\end{array}$ \\
\hline EB Kindler (EBK) & $\begin{array}{l}\text { Descrita mais recentemente, apresenta um quadro misto das outras formas anteriores e } \\
\text { as bolhas podem se formar em qualquer nível da derme, entre a lâmina lúcida e a lâmina } \\
\text { densa. Apresenta bolhas, sensibilidade ao sol, atrofia de pele, inflamação no intestino e } \\
\text { estenose de mucosas. }\end{array}$ \\
\hline
\end{tabular}

Fonte: Autores (2021).

As classificações da EB atuais baseiam-se principalmente no nível anatômico em que a divisão que forma a vesícula ocorre. Além disso, os achados clínicos, como cicatrizes, e herança são consideradas. As principais formas são definidas pelo nível da bolha e pela sua localização da pele. A epidermólise bolhosa ocorre em todas as raças e igualmente todos os sexos (Fantauzzi et al. 2008; Maschino et al. 2013).

A frequência e gravidade das manifestações bucais da epidermólise bolhosa podem variar de acordo com cada tipo de doença e podem ser confundidas com doenças como pênfigo, penfigóide bolhoso, dermatite herpetiforme, porfiria cutânea tardia, cútis aplástica, epidermólise bolhosa adquirida, impetigo, ou Síndrome de Stevens-Johnson (Scheidt et al., 2015). Diante desse contexto, o presente estudo possui como objetivo revisar a literatura acerca das manifestações orais encontradas em pacientes portadores da Epidermólise Bolhosa, bem como descrever o manejo clínico odontológico a ser seguido em casos de EB.

\section{Metodologia}

Trata-se de uma revisão narrativa da literatura. A revisão de literatura permite a busca aprofundada dentro de diversos autores e referenciais sobre um tema específico, nesse caso, as manifestações orais e manejo odontológico em pacientes com epidermólise bolhosa (Pereira et al. 2018). 
A fim de que haja direcionamento na pesquisa delineou-se como questão norteadora: "Quais são as manifestações orais decorrentes da epidermólise bolhosa?" e "qual deve ser a conduta odontológica em pacientes que possuem essa condição?"

Para a construção deste artigo foi realizado um levantamento bibliográfico nas bases de dados SciVerse Scopus, Scientific Eletronic Library Online (Scielo), U.S. National Library of Medicine (PUBMED) e ScienceDirect, com auxílio do gerenciador de referências Mendeley. Os artigos foram contemplados entre os anos de 2010 a 2021.

A estratégia de pesquisa desenvolvida para identificar os artigos incluídos e avaliados para este estudo baseou-se nos descritores contidos na lista dos Descritores em Ciência da Saúde (DeCS) e suas combinações no idioma português e inglês: [(epidermólise bolhosa OR bullous epidermolysis) AND (mucosa oral OR oral mucosa OR boca OR mouth OR mucosa jugal OR cheek mucosa OR língua OR tongue) AND (manifestações clínicas OR clinical findings OR tratamento OR treatmente OR etiologia OR etiology OR diagnóstico OR diagnosis)]

\subsection{Critérios de inclusão e exclusão}

Considerou-se como critério de inclusão os artigos completos disponíveis na íntegra nas bases de dados citadas, nos idiomas inglês e português e relacionados com o objetivo deste estudo. Os critérios de exclusão foram artigos incompletos, duplicados, resenhas, estudos in vitro e resumos.

\subsection{Seleção de estudos}

A estratégia de pesquisa baseou-se na leitura dos títulos para encontrar estudos que investigassem a temática da pesquisa. Caso contemplasse esse primeiro objetivo, posteriormente, os resumos eram lidos e, persistindo na inclusão, era feita a leitura do artigo completo. Quando havia dúvida sobre a inclusão, o artigo era lido por outro autor e, a decisão de inclusão ou exclusão era tomada em consenso.

\section{Resultados e discussão}

Com base na revisão de literatura feita nas bases de dados eletrônicas citadas, foram identificados 830 artigos científicos, dos quais 148 estavam duplicados com dois ou mais índices. Após a leitura e análise do título e resumos dos demais artigos outros 628 foram excluídos. Assim, 54 artigos foram lidos na integra e, com base nos critérios de inclusão e exclusão, apenas 25 artigos foram selecionados para compor este estudo. O fluxograma com detalhamento de todas as etapas de seleção está na Figura 1. 
Figura 1 - Fluxograma de identificação e seleção dos estudos.

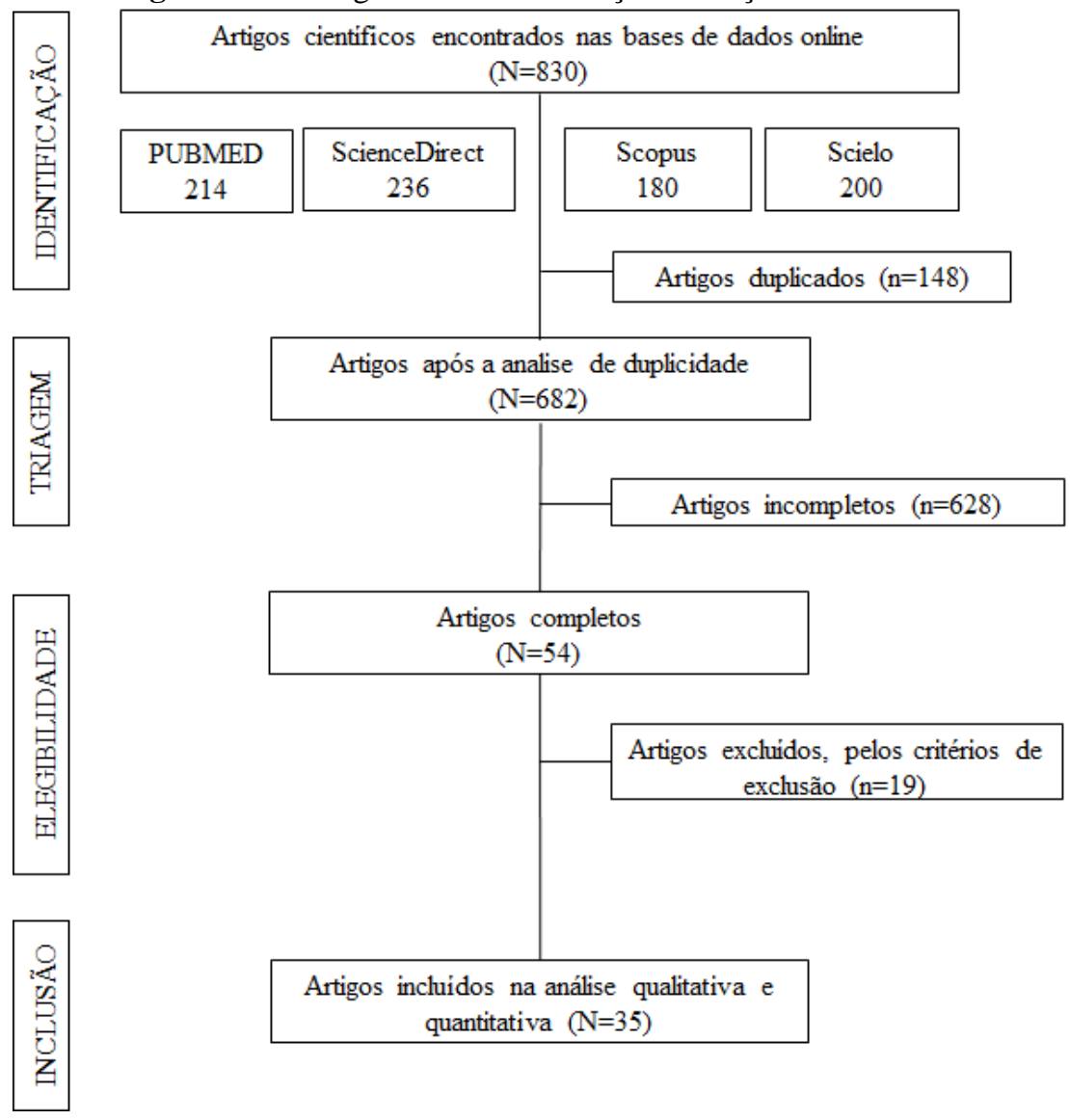

Fonte: Autores (2021).

Na cavidade oral, a EB pode manifestar-se de diversas formas, com inúmeras alterações, que variam em severidade e quantidade, dependendo do tipo de EB, como a formação de bolhas e ulcerações (na mucosa, palato e língua), anodontia, junção do sulco vestibular (união da gengiva inserida com a mucosa bucal), presença das doenças cárie e periodontal severas, reabsorção óssea alveolar e anomalias dentárias hipoplásicas, além de atrofia da maxila, prognatismo mandibular e ângulo da mandíbula aumentado. A maloclusão torna-se ainda mais severa pela atresia dos arcos alveolares e pelo atraso na erupção dentária (Oliveira 2018; Rezende et al. 2019)

As principais manifestações orais são: lesões bolhosas polimórficas nas regiões jugal e vestibular, vermelhidão nos lábios e nas formas juncional e distrófica há suscetibilidade de injúrias, como erosões e eventual desnudamento da língua, além da ocorrência de anquiloglossia e microstomia. A presença de lesões na cavidade bucal e faringe leva à disfonia (redução da abertura da boca), deglutição atípica, desequilíbrio da musculatura e alterações no desenvolvimento dos maxilares, extração dentária deve ser evitada nas formas graves, dispositivos ortodônticos favorecem o atrito com os tecidos bucais e a formação de bolhas e infecções (Chan et al. 2019).

A gravidade das manifestações orais relacionadas a EB podem variar de acordo com cada caso clínico. Ambos os tecidos moles e duros podem ser afetados pelas bolhas e, em alguns subtipos da doença, pode ocorrer hipoplasia do esmalte. A maioria dos pacientes acometidos pela epidermólise desenvolve lesões na cavidade oral em pelo menos uma vez na vida, com todos os tecidos moles afetados, ocasionando fragilidade na mucosa. Ademais, a literatura aponta grande frequência de queilite angular provocada por qualquer trauma mínimo, como ingestão de alimentos, higienização oral ou, ainda, pelo tratamento dentário (Czlusniak \& Schwab 2012). 
Além disso, foi constatado que a presença de bolhas seguida pela cicatrização pode interferir no crescimento maxilarmandibular, prejudicar o movimento da língua (anquiloglossia), microstomia, bliteração vestibular, perda das rugas palatinas e lingual papilas, apinhamento dentário e cárie dentária extensa devido à falta de higiene bucal. Inflamação crônica e a desnutrição também podem desempenhar um papel na despapilação lingual e mau posicionamento do dente (Branco 2010; Nazir \& Chalkoo 2017).

Com ciclos repetidos de formação de bolhas e cicatrização, funções orais como morder, mastigar, engolir e falar tornase reduzidos. Essas funções podem ser dolorosas e, consequentemente, a ingestão de alimentos (principalmente sólidos) pode ser prejudicada. A má prática de higiene oral resulta de a incapacidade de escovar os dentes, pois este procedimento é doloroso e evoca a formação de bolhas, o que pode levar a um início mais severo de cáries (Branco 2010; Nazir \& Chalkoo 2017).

\subsection{Manejo odontológico em pacientes com Epidermólise Bolhosa}

Para todos os casos de EB, deve-se incluir três estratégias principais: prevenção pela higiene bucal e redução do consumo de alimentos cariogênicos - medida de suma importância para minimizar o surgimento da cárie; restauração de defeitos de esmalte e dentina com restaurações e/ou coroas de aço inoxidável para garantir a estrutura e função dos dentes; e 14 extrações dos dentes mais afetados a fim de eliminar as fontes contínuas de infecções bucais. O selamento de fissuras e fossas é recomendado. Alguns clínicos especialistas, no entanto, têm apreensões em relação a este conselho, como a técnica é muito sensível e pode não ser uma opção para alguns pacientes por causa de cooperação, tendo acesso comprometido, é difícil acompanhamento em longo prazo (Branco 2010; Dağ et al., 2014; Nazir \& Chalkoo 2017).

Tabela 2 - Manejo odontológico em pacientes portadores de Epidermólise Bolhosa.

\begin{tabular}{|c|c|c|c|}
\hline EB Simples & EB Distrófica & EB Juncional & Síndrome de Kindler \\
\hline $\begin{array}{l}\text { O profissional deve perguntar sobre } \\
\text { a história de fragilidade da mucosa, } \\
\text { porque a manipulação pode } \\
\text { precipitar lesões em pacientes } \\
\text { levemente afetados; } \\
\text { A anestesia é a técnica de escolha. } \\
\text { Higiene oral e profilaxia adequadas } \\
\text { (uso de gazes e cotonetes). } \\
\text { As drogas tais como os } \\
\text { corticosteroides e outros agentes } \\
\text { antiinflamatórios podem ser usados } \\
\text { tópica ou oral, ajudam a controlar a } \\
\text { dor e coceira. } \\
\text { Se a candidíase elevar, os agentes } \\
\text { anti-candidal podem ser úteis. } \\
\text { Uso de Pomadas e géis que causam } \\
\text { dormência local e aliviam a dor, } \\
\text { como cloridrato de lidocaína }\end{array}$ & $\begin{array}{l}\text { Realizar exercícios diários para } \\
\text { melhorar/ manter uma boa abertura } \\
\text { de boca. Isso pode ser realizado, por } \\
\text { exemplo, durante as trocas de } \\
\text { curativos. } \\
\text { Melhorar a abertura da boca também } \\
\text { favorece fonação e deglutição. A } \\
\text { realização de exercícios meia hora } \\
\text { antes tratamento dentário ajuda a } \\
\text { melhorar o acesso; } \\
\text { Prescrições medicamentosas devem } \\
\text { estar na forma solúvel ou líquida. } \\
\text { Pais }\end{array}$ & $\begin{array}{l}\text { O tratamento dentário não requer } \\
\text { muitas modificações; no entanto, } \\
\text { uma cuidadosa abordagem é } \\
\text { recomendada na manipulação dos } \\
\text { tecidos que podem produzir } \\
\text { ulceração oral. } \\
\text { Requer um programa preventivo } \\
\text { agressivo e visitas frequentes ao } \\
\text { dentista, uma vez que se apresentam } \\
\text { com hipoplasia do esmalte, levando } \\
\text { a um aumento risco de cáries e } \\
\text { desgaste severo. } \\
\text { A anestesia Geral pode ser indicada } \\
\text { para alguns procedimentos } \\
\text { extensivos, em situações como: a } \\
\text { capacidade abrir a boca, dores fortes, } \\
\text { presença de muitas bolhas ou que o } \\
\text { tratamento a ser realizado é muito } \\
\text { complexo }\end{array}$ & $\begin{array}{l}\text { Pessoas afetadas pela síndrome de } \\
\text { Kindler costumam ter problemas } \\
\text { com a dentes (esmalte dentário e } \\
\text { cavidades) e / ou nas membranas } \\
\text { mucosas da cavidade oral. O } \\
\text { paciente com esta forma de EB tem } \\
\text { cárie excessiva que pode levar a uma } \\
\text { perda prematura de dentes. Bolhas e } \\
\text { erosões nos lábios, são } \\
\text { As gengivas também são } \\
\text { frequentemente afetadas devido à } \\
\text { inflamação crônica do mesmo } \\
\text { (gengivite) e deterioração mecânica } \\
\text { devido a cuidados com os dentes } \\
\text { necessários. } \\
\text { Será necessário fazer obturações ou } \\
\text { mesmo extrair dentes. Essas } \\
\text { intervenções terão que ser feitas } \\
\text { muitas às vezes sob anestesia total; } \\
\text { Escovação adequada, alimentação } \\
\text { saudável, com check-ups regulares } \\
\text { no dentista, bem como higiene } \\
\text { dental profissional, e higiene oral } \\
\text { diária em casa com uso de flúor, uso } \\
\text { de clorexidina, géis e selagem de } \\
\text { fissuras e recentemente uso de pastas } \\
\text { a base de cálcio e fosfato. }\end{array}$ \\
\hline
\end{tabular}

Fonte: Krämer et al. (2012).

A frequência da ida ao consultório odontológico deve ser realizada de acordo com cada caso, levando em consideração à quantidade de biofilme apresentada e risco de cárie dentária. As consultas devem ser direcionadas à prevenção de cárie e seu diagnóstico precoce, remoção do biofilme, aplicação de flúor, aconselhamento dietético, revisão do progresso ou deterioração da condição bucal do paciente (Valéria et al. 2007). 
Algumas estratégias de preventivas de higiene oral devem ser tomadas em pacientes que possuem EB. Em primeiro plano, a maior preocupação possui relação com possíveis danos que à mucosa oral causado pelo uso de escovas de dente. Nesse caso, algumas medidas podem ser tomadas para ajudar a escovação, como selecionar uma escova de cabeça pequena e com cedas macias (as cedas podem ser ainda mais amolecidas por imersão em água morna ou quente), as cedas podem ser cortadas para ajustar à abertura bucal em pacientes com microstomia de moderada a grave, também podem ser realizados ajustes no cabo da escova para pacientes com problemas de pseudo-sindactilia e destreza manual e, por fim, cotonetes, mini escovas descartáveis, pano de algodão limpo ou gaze podem ser usados para limpar os dentes caso o paciente não consiga escovar temporariamente devido a lesões causadas pela doença (Branco 2010; Galeotti et al. 2014; Nazir \& Chalkoo 2017; Valéria et al. 2007).

\section{Considerações Finais}

A partir das trajetórias bibliográficas dos pesquisadores, esse estudo abordou as principais manifestações orais encontradas em pacientes acometidos pela epidermólise bolhosa, destacando o manejo odontológico a ser seguido nesses casos.

Devido à grande dificuldade e risco de se oferecer tratamento odontológico aos pacientes com EB, a prevenção da cárie, biofilme e da doença periodontal deve ser enfatizada desde a infância. Devendo o profissional no tratamento realizar higiene oral e profilaxia adequada, um programa dietético de prevenção de cárie, uso de géis, enxaguantes bucais, terapias adjuvantes com clorexidina D, cotonetes, sprays e aplicações de verniz tópico, fluoretos, raspagem de biofilme e lazer.

Com isso, o levantamento bibliográfico sobre as manifestações orais encontradas na EB se mostra importante, pois envolve estudos retrospectivos que possuem relação com o tema proposto e, ao mesmo, tempo, cria-se a possibilidade futura de novos pesquisadores abordarem o tema, criando novos trabalhos, para que haja melhora no manejo odontológico ofertado a esses pacientes, melhorando seu prognóstico e em sua qualidade de vida.

\section{Referências}

Angelo, M. M. F. C., Diurianne, C. de C. F., Daniely, B. R. do L., \& Luiz, E. R. V. (2012). "Manifestações Clínicas Da Epidermólise Bolhosa: Revisão de Literatura." Pesquisa Brasileira Em Odontopediatria e Clinica Integrada 12(1):135-42.

Bega, A. G., et al. (2015). "Epidermólise Bolhosa: Revisão Da Literatura." 7-10.

Branco, J. De F. (2010). “Terapia Nutricional Na Epidermólise Bolhosa Simples.” 25(3):251-55.

Chan, J. M., et al. (2019). “Occupational Therapy for Epidermolysis Bullosa: Clinical Practice Guidelines.” Orphanet Journal of Rare Diseases 14(1):129.

Czlusniak, Gislaine Denise, and Carolina Barbosa Schwab. 2012. "Epidermólise Bolhosa Distrófica Recessiva Generalizada: Protocolo de Atendimento Odontológico e Relato de Caso Recessive Generalized Dystrophic Epidermolysis Bullosa : Dental Service Protocol and Case Report.” 47(4):237-43.

Dağ, Canan, Tuğba Bezgin, and Nurhan Özalp. 2014. "Dental Management of Patients with Epidermolysis Bullosa.” Oral Health and Dental Management 13(3):623-27.

Fantauzzi, R. et al. (2008). "Manifestações Otorrinolaringológicas e Esofágicas Da Epidermólise Bolhosa.” 74(5):657-61.

Galeotti, A., et al. (2014). "Er:YAG Laser Dental Treatment of Patients Affected by Epidermolysis Bullosa.” Case Reports in Dentistry 2014:421783.

Maschino, F., J. Guillet, R. Curien, G. Dolivet, P. \& Bravetti. (2013). “Oral Metastasis: A Report of 23 Cases.” International Journal of Oral and Maxillofacial Surgery 42(2):164-68.

Nazir, N., \& Altaf, H. C. (2017). “Oral Manifestations of a Patient with Epidermolysis Bullosa.” 1(6):1562-65.

Oliveira, P. C. S. De. (2018). “Condição e Cuidados de Saúde Bucal Em Portadores de Epidermólise Bolhosa: Relato de Casos.”

Pereira, A., et al. (2018). Método Qualitativo, Quantitativo Ou Quali-Quanti.

Pitta, A. L., et al. (2016). “Enfermagem Congenital Epidermolysis Bullosa - Importance Of Nursing Care.” 10(2):201-8.

Rezende, R. P., Naiara, S. R., Patricia, M., \& Leite R. (2019). "Manifestações Bucais Da Epidermólise Bolhosa: Relato de Caso.”

Scheidt, L., et al. (2015). “Oral Manifestations and Dental Management of Epidermolysis Bullosa Simplex.” International Journal of Clinical Pediatric Dentistry $8(3): 239-41$.

Valéria, P., et al. (2007). “Atendimento Multidisciplinar Do Paciente Ortodôntico Com Epidermólise Bolhosa.” 30-35. 